Última DÉCADA N4, ProyeCTO JUVENTUDES, DiCIEMBRE 2016, PP. 93-116

\title{
EL MOVIMIENTO ESTUDIANTIL CHILENO (2006-2016) Y EL USO DE LA WEB SOCIAL: NUEVOS REPERTORIOS DE ACCIÓN E INTERACCIÓN COMUNICATIVA
}

\author{
CAMila CÁRDENAS NEIRA*
}

\begin{abstract}
RESUMEN
En este artículo analizamos dos dimensiones distintivas del movimiento estudiantil chileno (2006-2016): 1) la reconfiguración de los repertorios de acción juvenil soportados tecnológicamente, y; 2) la conformación de nuevos repertorios de interacción comunicativa en redes sociales. Primero proponemos una aproximación multidisciplinar que nos ayude a responder cómo los/as estudiantes modifican la estructura de oportunidades mediáticas al posicionar sus luchas en el espacio público. Posteriormente aportamos un modelo teóricoanalítico que nos permita abarcar dichos cambios, atendiendo los procesos de construcción de significados y las prácticas discursivas desarrolladas en la Web Social. Concluimos que la convergencia de ambos repertorios impacta simultáneamente a un nivel socio-cognitivo, socio-semiótico y político-histórico, con rendimientos materiales y simbólicos que pocos estudios han integrado hasta la fecha.
\end{abstract}

Palabras Clave: Movimiento Estudiantil Chileno, Repertorios De (InTer)Acción, WEB SOCIAL.

\section{O MOVIMENTO ESTUDANTIL CHILENO (2006-2016) E O USO DA WEB SOCIAL: NOVOS REPERTÓRIOS DE AÇÃO E INTERAÇÃO COMUNICATIVA}

\begin{abstract}
RESUMO
Este artigo analisa duas dimensões distintivas do movimento estudantil chileno (20062016): 1) a reconfiguração dos repertórios de ação juvenil tecnologicamente suportados, e; 2) a criação de novos repertórios de interação comunicativa nas redes sociais. Primeiro, propomos uma abordagem multidisciplinar para nos ajudar a responder como os e as estudantes mudam a estrutura de oportunidades que dá a mídia para posicionar suas lutas na esfera pública. Depois, nós entregamos um modelo teórico e analítico, que nos permite abordar estas mudanças considerando os processos de construção de significados e práticas discursivas desenvolvidas na Web Social. Concluímos que a convergência de ambos os repertórios atinge, simultaneamente, um nível sócio-cognitivo, sócio-semiótico e políticohistórico, com rendimentos materiais e simbólicos que poucos estudos integraram até agora.
\end{abstract}

PALAVRAS-CHAVE: MOVIMENTO ESTUDANTIL CHILENO, REPERTÓRIOS DE (INTER)AÇÃO, WEB SOCIAL.

\footnotetext{
* Doctoranda en Traducción y Ciencias del Lenguaje y miembro del Grupo de Estudios del Discurso (GED) de la Universitat Pompeu Fabra, Barcelona, España. Correo electrónico: camila.cardenas.neira@gmail.com
} 
Última DÉCADA N4, ProyeCTO JUVENTUDES, DiCIEMBRE 2016, PP. 93-116

\title{
THE CHILEAN STUDENT MOVEMENT (2006-2016) AND THE USE OF THE SOCIAL WEB: NEW ACTION AND COMMUNICATIVE INTERACTION REPERTOIRES
}

\begin{abstract}
This article analyzes two distinctive dimensions of the Chilean student movement (20062016): 1) the reconfiguration of technologically supported youth action repertoires, and 2) the creation of new communicative interaction repertoires in social networks. First, we propose a multidisciplinary approach to give us insight on the way students change the structure of media opportunities by means of positioning their struggles into the public space. Later, we bring a theoretical analytical model that allows us to embrace these changes, dealing with the processes of construction of meaning and discursive practices developed in the Social Web. We conclude that the convergence of both repertoires have a simultaneous impact on the socio-cognitive, socio-semiotic and political-historical levels, with material and symbolic yields still unattended enough up to date.
\end{abstract}

KEYWORDS: CHILEAN STUDENT MOVEMENT, (INTER)ACTION REPERTOIRES, SOCIAL WEB.

\section{NUESTRO ESTUDIO}

Este trabajo se desprende de una investigación más amplia (Cárdenas, 2016, 2014a, 2014b, 2014c, en prensa) que se plantea estudiar los discursos publicados en Facebook en el contexto de las últimas movilizaciones estudiantiles en Chile (2011-2013). Nuestro objetivo general consiste en averiguar cómo los/as estudiantes producen, transponen e interpretan significados sobre el conflicto educativo, a partir de su instanciación en diversos géneros textuales, modos expresivos y soportes mediales. Nuestras indagaciones actuales pretenden abordar estos procesos en relación con tres espacios que resultan constitutivos de la acción juvenil posdictatorial: las calles, las aulas y las redes sociales (Cárdenas, 2014b), enfocándonos en la compleja interacción entre prácticas sociales, espaciales y comunicativas (Martín Rojo, 2016a).

Con este artículo intentaremos mostrar que la articulación de espacios públicos y mediáticos es esencial para explicar: 1) los alcances e impactos del movimiento estudiantil reciente, en general, y; 2) los usos situados y estratégicos de la Web Social por parte de sus miembros, en particular. Para ello privilegiaremos una aproximación que pone énfasis en otros factores menos estudiados, como son sus rendimientos semióticos y cognitivos. A continuación, presentamos una discusión bibliográfica que nos ayudará a sintetizar cómo se ha tratado hasta la fecha el vínculo entre movimientos sociales y nuevas tecnologías. Luego, desarrollamos dos apartados en los que analizaremos la reconfiguración de las prácticas colectivas estudiantiles, cuyas innovaciones ponderaremos diacrónicamente (2006-2016). Por último, precisamos nuestra metodología de trabajo y proponemos un modelo teórico-analítico provisorio a partir de nuestros primeros hallazgos, con el que buscaremos dar cuenta de las principales prácticas discursivas que los/as jóvenes movilizados/as llevan a cabo en sus comunidades online, sobre la base de sus procesos de significación más prominentes. 


\section{Preliminares teóricos}

Si bien últimamente han proliferado investigaciones que buscan explicar los rasgos distintivos de los actuales movimientos sociales en red (Feixa, Fernández-Planells y Figueras-Maz, 2016; Castells, 2014, 2012; Bennett y Segerberg, 2013; Tarrow, 2011; Tilly y Wood, 2010; Feixa, Pereira y Juris, 2009), son todavía pocos los estudios que han reparado en el papel de los medios y las prácticas de comunicación (Costanza-Shock, 2014, 2013, 2012; Cammaerts, Mattoni y McCurdy, 2013; Fernández-Planells, Feixa y FiguerasMaz, 2013; Castells, 2009, 2007) más allá de su carácter logístico o utilitario, dando alguna cabida a los procesos de elaboración, circulación y comprensión que configuran determinados discursos de protesta (Cárdenas, 2016, 2014a, 2014b; Martín Rojo, 2016b, 2013; Georgalou, 2015; Montesano y Morales, 2015; Romano, 2015; García y Aguirre, 2016; Sierra, 2012).

La producción académica sobre el rol desempeñado por internet y redes sociales en diversas movilizaciones globales aumenta exponencialmente en las dos últimas décadas, proponiendo diversas etiquetas que abarcan las posibles relaciones entre tecnología y acción colectiva: clickactivismo (Peña-López, 2013; Butler, 2011), hacktivismo (Jordan y Taylor, 2004; Samuel, 2004), ciberactivismo (Tascón y Quintana, 2012; McCaughey y Ayers, 2003), ciberprotesta (van de Donk et al., 2004; Pickerill, 2003), ciberpolítica (Bell, 2006; Hill y Hughes, 1998) y tecnopolítica (Toret, 2015; Toret et al., 2013) son algunas de las más frecuentes. Sus rasgos a menudo se yuxtaponen, y acaban formando parte de un continuum que implica una comprensión más o menos compleja de las prácticas sociotécnicas involucradas.

De un lado, identificamos una aproximación que tiende a disociar los ámbitos de sociabilidad online y offline, de modo que al comparar el impacto de las actividades gestadas dentro y fuera de la web se polarizan sus beneficios y limitaciones. En este caso el énfasis está puesto en cómo los usos tecnológicos traducen los rendimientos políticos, evaluándose su nivel de dependencia y eficacia. De otro lado, identificamos una aproximación que propende a la integración de los espacios de acción social e interacción comunicativa, los cuales se articulan estructuralmente a partir de las conexiones que los colectivos y sus miembros motivan con base en sus experiencias cotidianas. En este caso, el énfasis está puesto en cómo los rendimientos políticos subsumen los usos tecnológicos, evaluándose su nivel de interdependencia e influencia.

En este escenario, nuestra postura adhiere a la propuesta de Sasha Costanza-Shock (2014), para quien no resulta provechoso tratar de probar o refutar una relación causal entre ambos componentes, sino más bien examinar las formas en que los medios de comunicación son en realidad parte integrante de la construcción de los movimientos. En esta línea, pensamos que puede ser más productivo concentrarnos en cómo los movimientos utilizan las tecnologías, con el propósito de esclarecer las funciones políticas que dichos usos comunicativos cumplen en contextos específicos. Parte de la literatura que hemos revisado hasta ahora nos ha permitido observar que tales funciones involucran dimensiones materiales y simbólicas que no siempre han sido articuladas ni concebidas en forma interdependiente. 
De esta manera, se han disgregado los usos que apuntan a funciones que llamaremos provisionalmente pragmáticas, como son organizar eventos masivos, establecer tácticas de ocupación y resistencia contra la represión policial y la vigilancia gubernamental, y obtener información acerca de las protestas en curso y otras coyunturas políticas influyentes; de los usos que apuntan a funciones que llamaremos provisionalmente semánticas, como son identificarse con el endogrupo y diferenciarse del exogrupo, reflexionar acerca de los asuntos que resultan relevantes para asegurar la cohesión grupal y el decurso de las movilizaciones, y debatir sobre los lineamientos ideológicos que enmarcan las estrategias de interpretación y acción colectiva.

Mientras que las funciones pragmáticas se orientan a propósitos más bien concretos, de gestión de contacto e interacción, las funciones semánticas se orientan a propósitos más bien abstractos, de co-construcción de sentidos e intersubjetividad. Es evidente que ambas dimensiones se activan y se afectan mutuamente, pero en muchos casos las investigaciones han tendido a privilegiar una en lugar de la otra en virtud de sus tradiciones y énfasis disciplinares, simulando una fragmentación artificiosa (Mattoni, 2013).

Para lograr una comprensión integrada de estos aspectos, proponemos avanzar a través de dos constructos teóricos: desde los repertorios de acción política a los repertorios de interacción comunicativa. Dicho tránsito sugiere, al menos como tentativa inicial, un subsecuente desplazamiento desde las estructuras de oportunidades políticas a las estructuras de oportunidades mediáticas. El propósito que orienta este movimiento consiste en esclarecer los puntos de convergencia entre ambos tipos de repertorios y sus rendimientos recíprocos. Según Mattoni, estos se encuentran interrelacionados toda vez que "la elección de actuaciones contenciosas específicas implica ciertas prácticas mediáticas activistas y éstas, a su vez, también afectan las actuaciones contenciosas en sí mismas" (2013: 50). De hecho, Gamson y Wolfsfeld (1993) plantean que algunas de las oportunidades y constricciones políticas son inherentes al funcionamiento mismo de los medios de comunicación.

De acuerdo a McAdam, McCarthy y Zald (1999), existe consenso acerca de la incidencia que los sistemas políticos institucionalizados tienen sobre las posibilidades de acción colectiva y las formas que esta adopta en virtud de las variables que intervienen en la constitución de actores y redes. En esta dirección, la emergencia de los repertorios incluye diversas modalidades de acción coordinada dependientes de estas coyunturas, las cuales responden a una estrategia de consecución de objetivos comunes supeditada a un contexto y una estructura de movilización particulares. En palabras de Tilly, los repertorios de acción equivalen a:

un conjunto limitado de rutinas adquiridas, compartidas y actuadas a través de un proceso de elección relativamente deliberado. Los repertorios son creaciones culturales aprendidas, pero no descienden de la filosofía abstracta ni toman forma como resultado de la propaganda política, sino que surgen de la lucha. Es en la protesta donde la gente aprende a romper ventanas, atacar presos sujetos al cepo, derribar casas deshonradas, escenificar marchas públicas, hacer peticiones, mantener reuniones formales u organizar asociaciones de intereses especiales. (2002: 31) 
Siguiendo a este autor, un repertorio puede ser entendido como un lenguaje, en tanto las acciones que lo componen siempre conectan conjuntos de individuos y grupos, quienes siguen unas pautas recurrentes y familiares y trazan un horizonte de experiencia colectiva gracias a la comprensión, los recuerdos y los acuerdos que comparten. Además, estas acciones "no son sólo lo que hace la gente cuando entra en conflicto con otros; es lo que sabe hacer y lo que los otros esperan que haga" (Tarrow, 2011: 66, cursiva en el original). De este modo, los repertorios no sólo se expanden o diversifican con el tiempo, sino que también se resignifican, esto es, activan nuevos excedentes de sentido según sean sus trayectorias propias y distintivas.

Según Costanza-Shock $(2013,2012)$, la rápida innovación de las tecnologías y las prácticas de comunicación en redes sociales nos obliga a considerar la noción de estructura de oportunidades mediáticas de manera complementaria a la noción de oportunidades políticas, ya que, en el escenario actual, resulta ineludible atender la capacidad de los movimientos sociales de lograr visibilidad y cohesión grupal elaborando formas cotidianas de contención y contestación online. Al respecto, destaca el aprovechamiento de las herramientas digitales por parte de los actores movilizados con el fin de ejercer presión horizontal sobre los flujos informativos que suelen organizarse verticalmente, de modo de tensionar los intereses de la clase poderosa comprometida con el manejo de los grandes consorcios periodísticos.

En esta línea, los movimientos no sólo aspiran a aumentar su grado de influencia en la esfera pública ingresando en la agenda de los medios de comunicación hegemónicos; ellos, sobre todo, integran el uso de medios alternativos y propios para conseguir mayor apoyo político, incrementar la legitimación de sus demandas y extender los alcances del conflicto por fuera de sus dominios (Mattoni, 2013). Para lograr estos objetivos, sus miembros articulan una estructura de oportunidades de mediación más amplia, la cual se compone tanto de las oportunidades mediáticas aludidas, como de las oportunidades discursivas y las oportunidades de las propias redes sociales (Cammaerts, 2012). Con esta estructura podemos distinguir entre los diferentes actores y sus formas de organización, la utilización de diversos formatos textuales y marcos ideológicos, y las múltiples estrategias de auto-mediación facilitadas por tales plataformas.

Estos planteamientos otorgan centralidad a la noción de repertorios comunicativos, los cuales abarcan "todo el conjunto de las prácticas mediáticas activistas que los (...) movimientos sociales podrían concebir como siendo posibles y desarrollándose (...) en las fases latentes y visibles de la movilización, para llegar a los actores ubicados tanto dentro como fuera de su entorno" (Mattoni, 2013: 47). De acuerdo a esta autora, estas prácticas se subdividen en prácticas de conocimiento medial, que aluden a la construcción de saberes específicos sobre el ecosistema mediático donde el movimiento se desenvuelve, y prácticas mediales relacionales, que remiten a la interacción con otros medios, empresas y profesionales. En adelante, privilegiamos la noción de repertorios de interacción comunicativa para abarcar las dimensiones epistémica y dialógica de las prácticas discursivas desarrolladas por los actores movilizados en la Web Social.

En síntesis, estos repertorios son situados, pues se insertan en un contexto concreto que proporciona a los movimientos sociales unos recursos logísticos y expresivos particulares cuyos usos se adaptan en función de sus respectivas culturas políticas e históricas; y son dinámicos, pues son sensibles al ambiente y a los adelantos tecnológicos 
emergentes. Según Mattoni (2013), con esta noción podemos aproximarnos a tres dinámicas político-mediáticas interrelacionadas: transformaciones en la visibilidad del movimiento, cambios en sus patrones de reconocimiento y variaciones en la formación de identidades de sus miembros. Estos últimos aspectos, entre otros afines, son los que analizaremos en las secciones siguientes.

\section{EL MOVIMIENTO ESTUDIANTIL CHILENO DE POSDICTADURA}

Uno de los principales desafíos del movimiento estudiantil chileno consiste en reconstituirse tras el término de la dictadura cívico-militar (1973-1990), al alero de una sociedad que sobrelleva un importante proceso de desmovilización entre los años 90 y mediados de los 2000, fruto de las experiencias traumáticas que deja el terrorismo de Estado. De allí deriva la necesidad de adecuar sus repertorios de acción al escenario postransicional, caracterizado por la preeminencia de un discurso y una práctica que pretendieron desconectar de la política a la ciudadanía en su conjunto (Muñoz, 2011), de un lado, y por la pervivencia de un aparato de disposiciones constitucionales y enclaves autoritarios que condiciona varios derechos fundamentales y exacerba lógicas insoslayables de segregación y desigualdad (Garretón et al., 2011), del otro.

Por lo anterior, los/as estudiantes buscan reposicionarse públicamente en una coyuntura multidimensional que es transversal a su reciente ciclo movilizatorio (20062016): la crisis del modelo neoliberal (Aguirre y García, 2015; Bellei, Cabalin y Orellana, 2014; Bellei y Cabalin, 2013; Latorre, 2013; Cabalin, 2012; Mayol, 2012; Mayol y Azócar, 2011). De esta forma, el movimiento asume en la última década la posición de un universal polémico, pues en tanto "reinsertan el conflicto y el disenso como parte natural de la dinámica social, sus demandas no queden restringidas únicamente al ámbito educativo. La crítica a la privatización de la educación deviene así en una crítica a la democracia consensual" (Aguirre y García, 2015: 160).

Como veremos, la ubicación de los/as estudiantes en un tiempo y lugar determinados condiciona sus tácticas de protesta en virtud de las vivencias, lecciones y memorias acumuladas, por un lado, y de ciertos órdenes estructurales que demandan ejercicios de reinvención y resistencia sensibles a las disputas permanentes por el poder, por el otro. En los próximos apartados desarrollaremos estos alcances con mayor profundidad.

\section{a) Repertorios de acción colectiva}

De acuerdo a Melucci, "la "novedad" es por definición un concepto relativo que tiene la función temporal de señalar algunas diferencias comparativas entre las formas históricas del conflicto (...) y las formas emergentes de acción colectiva" (1994: 123). Desde esta perspectiva, el movimiento estudiantil de posdictadura no sólo experimenta transformaciones a propósito del cambio epocal y generacional que involucra su emplazamiento y recomposición en democracia, sino que, a su vez, conjuga dichos factores con otros de tipo técnico y comunicativo que delimitan sus medios y modos de expresión y visibilización en la última década (2006-2016).

Planteamos que una de las principales variables que interviene en estos cambios es la convergencia de espacios públicos y mediáticos que los/as jóvenes (re)apropian 
simultáneamente para dar consistencia y continuidad a su actuación grupal (Cárdenas, 2014b). Por ello, la concepción de novedad estaría íntimamente ligada a las alteraciones que repercuten en la escenificación del conflicto educativo (Aguilera, 2014), lo que hace a los/as estudiantes transitar de modalidades de contestación tradicionales y centralizadas (i.e. la marcha en el centro de la ciudad, la ocupación de los recintos educativos), a modalidades moleculares e interconectadas (i.e. los foros de deliberación online, los grupos de interés en redes sociales).

En este contexto, asumimos que el uso de la Web Social es esencial para mediar la organización colectiva (Cammaerts, Mattoni y McCurdy, 2013) y fomentar maneras más espontáneas y colaborativas de hacer y estar juntos (García y Aguirre, 2016), en razón de lo cual se conformaría una compleja red de (inter)acciones que, sugerimos, puede leerse a la luz de los procesos semióticos que la constituyen, relevando sus funciones cognitivas y políticas. Si, como anticipábamos con Tilly (2002), los repertorios "pueden ser entendidos como un lenguaje (...), se requiere conocer las gramáticas que ordenan las acciones y que tienen la capacidad de afectar a otros" (Aguilera, 2014: 50, cursiva nuestra). Dicha metáfora implicaría la existencia de una estructura subyacente sujeta a unos principios de significación compartidos por la comunidad de referencia, los cuales dotarían de sentido las agencias y los intercambios juveniles.

Según Fernández (2013), los repertorios utilizados en las manifestaciones estudiantiles se han diversificado desde acciones más convencionales como marchar, gritar cánticos y consignas, llevar banderas y pancartas, a acciones de carácter más creativo que incluyen pasacalles con grupos musicales, improvisaciones dramáticas, cuerpos pintados, muñecos gigantes, máscaras y disfraces, etc. Todo esto impacta en los modos de ritualización adoptados por el movimiento (Aguilera, 2010a), los cuales se coordinan en "un continuum entre afirmación identitaria y conflicto social" (Aguilera, 2014: 38). Así, mientras los/as jóvenes "denuncian, imputan y llaman a la acción, asimismo encarnan, entretienen, [y] satirizan (...). De esta forma, ellos/as apelan a la conciencia y la emoción con miras a facilitar la comprensión de lo que, de otra manera, podrían ser ideas o ideales muy abstractos" (Pino-Ojeda, 2014: 135).

La renovación de estos repertorios no sólo ha incidido en la constitución de enclaves distintivos de socialización e identificación colectiva juvenil en la historia reciente. También responde a los modos hegemónicos con que los/as jóvenes son representados/as en la esfera pública y los medios de comunicación tradicionales, los que habitualmente persiguen instalarlos/as en un lugar subordinado dentro del debate político, restándoles legitimidad (Cárdenas, 2014a, en prensa). Por lo tanto, la concreción de protestas masivas, festivas y pacíficas busca confrontar los múltiples dispositivos de control que promueven una hetero-representación negativa del movimiento estudiantil, con el objetivo de resistir la marginación y denunciar la criminalización de su acción (Gascón, 2016; Aguilera, 2014; Cárdenas, 2014d).

En este punto, se torna evidente que los/as estudiantes han logrado combinar aspectos clásicos y emergentes de los nuevos movimientos sociales (García y Aguirre, 2016), inspirando una forma de movilización colectiva lúdica (Aguilera, 2010b) que fusiona sus luchas históricas con la lógica del espectáculo (Cuadra, 2012). Específicamente, mediante 
la realización de "performances artísticas colectivas"1 (Somma, 2012: 306), los/as jóvenes acaban erigiendo un rico relato mediático, una puesta en escena cuadro por cuadro desde la cual avanzan "hacia la construcción de discursividades más complejas que van produciendo sentidos políticos sobre las propias prácticas" (Aguilera, 2010a: 85). Con ello, se conformaría una inédita cultura de la protesta que es, al mismo tiempo, una protesta desde la cultura (Cuadra, 2012). Esto es así,

en tanto (...) este movimiento ha asumido el papel de desvelar las manipulaciones del significado que son inherentes a este contexto [neoliberal], [por lo que] sus técnicas performativas son capaces de comunicar no sólo lo que se denota, sino también de evocar el poder multifacético (...) que solo está disponible en el lenguaje del arte. Es de esta manera que el actual movimiento estudiantil liderado en Chile manifiesta la agencia de la cultura y su capacidad de transformar la realidad socio-política. (PinoOjeda, 2014: 135)

Desde el momento en que las redes sociales se integran a estos repertorios emergentes, no sólo se optimizan los métodos de coordinación y escenificación colectiva, sino que se dinamiza un efecto multiplicador de las acciones de protesta online y offline, posibilitando tanto su extensión geográfica como temporal. A su vez, en distintas plataformas interactivas se evidencia que esta clase de acciones desencadena interesantes procesos de recontextualización (Cárdenas, 2014a, 2014c), esto es, de movilización de significados a partir de la circulación de distintos productos semióticos -fotografías, vídeos, etc.- en diferentes espacios -las calles, las aulas- y prácticas sociales -las marchas, las ocupaciones- (Cárdenas, 2014b).

A estas nuevas formas de utilizar el espacio público-mediático resultan transversales ciertos fenómenos de señalización de la protesta, tales como la personalización de los mensajes, su corporalización, fluidez y polifonía, y las prácticas de resemiotización y transmedialidad que hacen posible su propagación (Martín Rojo, 2016b, 2013). Todos ellos intervienen para desplegar una narrativa que ayuda a reenmarcar el conflicto entre estudiantes y gobierno, la cual es potenciada y distribuida gracias a la interconexión de modos y medios de comunicación variados (García y Aguirre, 2016; Cárdenas, 2014a). Con esto se influye en la construcción de referencias identitarias e ideológicas comunes dentro de la comunidad estudiantil movilizada, a partir del sentimiento de pertenencia que implica hacerse parte de una causa compartida.

Estas acciones, además, cristalizan rendimientos de orden generacional e histórico. Por un lado, cumplen con el objetivo de producir extrañamiento (Cabello, 2012), dada su producción por fuera de los modos adultos - consensuados y autorizados- de pensar y hacer política, haciendo que pierdan su carácter regulatorio, partidista e institucional. Por otro lado, reivindican el derecho de irrumpir en territorios que, hasta hace algunos años, habían permanecido deshabitados y clausurados para el quehacer ciudadano, rompiendo con la lógica de uso del espacio público propia de los años posteriores al fin de la dictadura

\footnotetext{
${ }^{1}$ Por ejemplo, en 2011 destacaron por su sofisticación e impacto comunicacional: la Genkidama por la Educación, el Thriller por la Educación, el Vamos a la Playa por la Educación, las 1800 Horas por la Educación, entre otras. Para revisar estos y otros eventos de protesta ver: http://www.cartografiadelamovilizacion.cl/
} 
(Fernández, 2013). Esto le permitiría a los/as estudiantes reconstruir sus memorias, relevando el excedente cognitivo y emocional de los lugares que recuperan para sí (García y Aguirre, 2016).

La reconfiguración de estos repertorios de acción colectiva da cuenta, en suma, de un aprendizaje acumulado que redefine los alcances -los espacios y las temporalidades- de la política adultocéntrica, dejando emerger otras tácticas, otras conexiones, otras gestas. Todas ellas al modelarse, coordinarse y mediatizarse en internet, devienen en épicas, reivindicando su visibilidad, adhesión e impacto público. Por tanto, lo realmente novedoso de dichos repertorios no se reconoce ni se explica sin aludir a un proceso de recomposición más general de la protesta juvenil en posdictadura (Cárdenas, 2014b: 70), que es permeable a las oportunidades políticas y mediáticas que surgen a lo largo del conflicto.

En síntesis, creemos que es un hecho que la convergencia de espacios públicomediáticos facilita los procesos de organización, resistencia y ocupación en sus dimensiones offline y online, consolidando hoy por hoy un ámbito de investigación que está marcando pauta en los movimientos sociales a nivel global. Si bien en el caso del movimiento estudiantil chileno la función que desempeña la Web Social en los últimos años (2011-2016) ya puede rastrearse en la Revolución Pingüina (2006), proponemos que su integración actual supone transformaciones sin precedentes en sus recientes repertorios de acción, al relevar el rol que adquiere la comunicación en el surgimiento de nuevas prácticas tecno-políticas (Cárdenas, 2016; Martín Rojo, 2016a).

\section{b) Repertorios de interacción comunicativa}

Las redes sociales han logrado consolidarse como contraesferas de representación, participación y deliberación (Arancibia, Sadlier y Montecino, 2016; Millaleo, 2011; Cárdenas, en prensa), generando y transformando varias de las modalidades de contención, contestación y visibilización operadas durante las recientes movilizaciones estudiantiles. Sus usos fluctúan desde propósitos eminentemente prácticos como convocar, coordinar e informar, a otros más bien figurativos como identificarse, reflexionar y debatir, satisfaciendo funciones políticas y cognitivas que se despliegan en un continuum pragmático-semántico como el que hemos sugerido inicialmente.

Según Portillo et al. (2012), ampliar los límites espaciales de la política implica emplear las redes sociales como un frente de combate de doble significación. En primer lugar, como espacio de constitución de las prácticas colectivas juveniles, lo que posibilita la toma de acuerdos entre diversos actores y la respectiva difusión de actividades. En segundo lugar, como un nuevo lugar de conflictividad y apropiación de recursos, en donde la circulación de información y la construcción de conocimiento conforman bienes valiosos para la movilización.

Tal como apunta Valderrama (2013), la propagación, la organización y la identificación fueron los objetivos concretos perseguidos por los/as estudiantes secundarios/as durante la Revolución Pingüina. La plataforma que obtuvo mayor uso y popularidad fue Fotolog (Costa, 2008; Domedel y Peña y Lillo, 2008), en conjunto con otras herramientas como la mensajería instantánea -a través de Messenger y telefonía móvil-, y los correos electrónicos. 
A grandes rasgos, estas plataformas resultaron útiles para: 1) presentarse y articular contactos, interactuando con sus pares e intercambiando información relevante sobre el desarrollo de las protestas a nivel local y nacional; 2) coordinar y divulgar actividades, aportando especialmente a la descentralización geográfica, de manera tal que establecimientos educativos de distintas latitudes pudieron acceder al estado de las movilizaciones a lo largo del país y ejecutar tácticas sincrónicas comunes, y; 3) identificarse y persuadir, motivando instancias de discusión para defender las reivindicaciones del movimiento, negociar las acciones más adecuadas para presionar al gobierno y denunciar la represión policial.

En este sentido, el manejo de distintos dispositivos y entornos online durante el 2006 "permitió a la comunidad estudiantil construir un contramedio donde se reconocían como (...) estudiantes movilizados alineados bajo una misma lógica comunicacional" (Valderrama, 2013: 133), ayudó a dar a conocer "sus argumentos al país y a las autoridades" (Condeza, 2009: 72), y resultó eficaz al "contribuir a la inserción de los estudiantes secundarios en el espacio público (...), así como al desarrollo de una identidad y una causa común" (Torres y Costa, 2012: 134). Si bien hay autores que dudan del carácter genuinamente estratégico de estos usos (Millaleo, 2011), lo cierto es que estos antecedentes impiden explicar el rol de las redes sociales en las movilizaciones posteriores en base a un mero determinismo tecnológico.

Lo sucedido a contar del 2011 permite identificar una transición en términos políticocomunicacionales, lo que responde a un proceso de maduración que sintetiza y evalúa las experiencias obtenidas en 2006 (Aguilera, 2014). De esta forma, las prácticas mediales estudiantiles se orientan reflexivamente a la afirmación de una voz alternativa que posiciona a unos sujetos críticos de los conflictos que tienen lugar en la sociedad chilena. En efecto, uno de los rendimientos más sobresalientes derivados de estos usos consiste en la construcción de un discurso sobre los/as estudiantes y desde ellos/as (Aguilera, 2014), esto es, la consolidación de una agencia comunicativa que no precisa de intermediarios para lograr posicionarse.

En esta línea, las funciones desempeñadas por las redes sociales durante la Revolución Pingüina no sólo adquieren características más densas y complejas, sino que, además: a) alcanzan masividad: aumenta la penetración tecnológica en la población, en particular en los segmentos juveniles, y comienzan a involucrarse otros actores sociales en el conflicto -potencialmente cualquiera que pueda conectarse a estas redes-; b) se transversalizan: traspasan la distancia - del centro a las regiones- y el tiempo -permanecen activas incluso en periodos de menor actividad y exposición pública del movimiento-, y ya no dependen únicamente de las jerarquías de las orgánicas estudiantiles, pasando de medios institucionales (Confederación de Estudiantes de Chile, Asamblea Coordinadora de Estudiantes Secundarios, etc.) a agrupaciones universitarias y secundarias de base, y; c) son transmediales: la arquitectura de estas redes facilita la conexión entre plataformas diversas y promueve un mejor aprovechamiento de los recursos semióticos disponibles (enlaces, imágenes, vídeos, etc.), lo que hace posible combinarlos de múltiples maneras de forma simultánea, dinámica y colaborativa.

Las investigaciones cuantitativas que asocian el uso de redes sociales a la acción política confirman que esta relación debe observarse como un indicador de un renovado tipo de participación ciudadana (Valenzuela, Arriagada y Scherman, 2014, 2012; 
Valenzuela, 2013). Según Valenzuela, "puede que los jóvenes estén desconectados de los ámbitos políticos tradicionales, como elecciones y partidos políticos, pero están conectados con la esfera pública, y lo están en parte gracias al uso que hacen de Facebook, YouTube y otras plataformas sociales" (2012: 20). En este sentido, consumir e intercambiar información online revela consecuencias positivas en la disposición de los/as jóvenes a involucrarse en asuntos de interés nacional, mediante actividades como conversar sobre problemáticas contingentes con familiares y amigos/as, participar en reuniones comunales y protestar (Scherman y Arriagada, 2012a, 2012b). Y si bien las redes sociales no plantean una reinvención total de las formas de movilización juvenil, sí se convierten en un espacio de amplificación de las mismas (Scherman, Arriagada y Valenzuela, 2014, 2013).

Las investigaciones cualitativas que analizan los contenidos publicados por cuentas de redes sociales son escasas aún, no obstante, recuperan orientaciones que ya hemos presentado. Un caso interesante lo constituye el estudio de Cabalin (2014) sobre el de Facebook de la Federación de Estudiantes de la Universidad de Chile (FECH), quien identifica entre los usos analizados: movilizar adherentes a través de la convocatoria a acciones de protesta, resaltar los logros, apoyos destacados y las manifestaciones masivas, y recordar quiénes son los adversarios del movimiento. También detecta usos orientados a informar las principales razones del conflicto estudiantil, cuestión que contribuye a "la construcción de los marcos interpretativos para la acción colectiva" (2014a: 30), y al establecimiento de "un "contra-framing" de los eventos noticiosos publicados por los medios tradicionales" (2014: 31), de modo que se recalcan los logros del movimiento, y se contrarrestan los discursos oficiales que los deslegitiman.

Los antecedentes aportados hasta aquí permiten evaluar en dos direcciones los usos de la Web Social entre 2006 y 2016. En primer lugar, los/as estudiantes amplían, innovan y profundizan sus repertorios de interacción comunicativa sobre la base de un aprendizaje sistemático que no está determinado por el solo incremento de recursos tecnológicos, sino que deviene de un proceso de reflexión política más detenido sobre las potencialidades de producir y difundir sus propios discursos. En segundo lugar, la prevalencia de estas plataformas y dispositivos no desplaza ni opone otros repertorios de acción colectiva, como las modalidades de escenificación que revisamos previamente, sino que las incorpora, sintetiza y expande, generando nuevos lugares de identificación, interrelación y subjetivación del conflicto educativo.

En este sentido, es un hecho que los espacios mediáticos (como las redes sociales) comienzan a mostrar una articulación paradigmática con otros espacios públicos constitutivos de la movilización estudiantil (como las calles y las aulas). Si sus usos desempeñan funciones políticas y cognitivas de carácter práctico (pragmático) y figurativo (semántico), es vital preguntarse por las dimensiones materiales, mentales y simbólicas que se articulan simultáneamente en la comunicación juvenil. Concretamente, nos proponemos responder en los apartados siguientes: ¿cómo son empleadas estas redes por una comunidad online específica?, y ¿cuáles aspectos teórico-analíticos resultan relevantes para explicar sus patrones de gestión, publicación e interacción?

\section{Metodología y CORPUS de estudio}


Los resultados que reportamos se derivan de una investigación más amplia cuyo objeto de análisis son las publicaciones registradas en la fan page de Facebook Universitario Informado $^{2}$ (UI) entre 2011 y 2013. El corpus lo recolectamos apoyados de procedimientos propios de la Minería de Datos (MD) (Baeza-Yates, 2009, 2005), disciplina de las Ciencias de la Información que se preocupa por recoger grandes volúmenes de datos y descubrir patrones en ellos. Mediante la programación informática de un recuperador (crawler) se obtuvieron 18.306 publicaciones, extracción que fue posible gracias a la Interfaz de Programación de Aplicaciones disponible en la biblioteca de la plataforma. Estos datos fueron almacenados, depurados y organizados de acuerdo a los metadatos disponibles sobre las interacciones de los/as usuarios/as (cantidad de me gusta, comentarios y compartir).

Una vez aplicados los criterios de selección relativos a la popularidad (15 publicaciones más compartidas por trimestre) y la pertinencia temática (aquellas exclusivamente relacionadas con el movimiento estudiantil), obtuvimos 56 entradas y 2.838 comentarios, datos que procesamos cuantitativamente mediante Visualización de Datos (VD) (Alcalde, 2015; Martig et al., 2003), generando un conjunto de nubes de palabras y gráficos. Las entradas fueron analizadas a partir de 8 categorías emergentes: género multimodal $^{3}$, propósito comunicativo, temas, participantes representados, acciones representadas, modos de representación grupal, recursos semióticos utilizados y autoría. Los comentarios relacionados fueron analizados a partir de 3 categorías: adscripción, referencia y recursos semióticos utilizados.

El corpus final quedó compuesto por: a) fotografías: 59\%; b) caricaturas: $9 \%$; c) afiches: 9\%; d) discursos murales: 8\%; e) memes: 6\%; f) videos: 5\%, y; g) noticias: 4\%. Estas publicaciones fueron procesadas cualitativamente con base en las aportaciones de los Estudios Críticos del Discurso (ECD) (Wodak, 2013; Van Dijk, 2011a; Van Leeuwen 2008; Fairclough, 1995), campo de investigación multi-disciplinario y multi-metodológico del cual adoptamos tres aproximaciones provenientes de las Ciencias del Lenguaje: la Lingüística Sistémico-Funcional (LSF) (Halliday y Matthiessen, 2014; Martin, 2014; Eggins, 2002), la Semiótica Social (SS) (Kress, 2010; Kress y Van Leeuwen 2006, 2001; Van Leeuwen 2005), y el Análisis Socio-Cognitivo del Discurso (ASCD) (Van Dijk, 2014, 2012, 2011b, 1999). A grandes rasgos, estas aproximaciones promueven el análisis de los usos situados del lenguaje, esto es, aquellas realizaciones lingüísticas y semióticas observadas en contextos económicos, políticos, culturales e históricos concretos donde resultan evidentes ciertas problemáticas de dominación o resistencia.

Nuestro modelo de análisis fue concebido para atender e integrar dos dimensiones: una socio-semiótica y otra socio-cognitiva, cada una de las cuales, a su vez, fue trabajada a un micro y macro nivel. Con el análisis socio-semiótico abordamos los patrones de construcción de: 1) significados composicionales: cómo son representadas las calles, las aulas y las redes sociales como espacios constitutivos de la acción política juvenil; 2) significados ideacionales: cómo son representados los participantes (miembros del movimiento estudiantil, aliados, adversarios, etc.) que se desenvuelven en dichos espacios y sus respectivas acciones en él, y; 3) significados interpersonales: cómo son representadas las relaciones (de poder, de solidaridad, etc.) que se establecen entre los participantes, y entre ellos y los espacios en los que interactúan. Con el análisis socio-cognitivo abordamos

\footnotetext{
${ }^{2}$ Se puede acceder en: https://www.facebook.com/UniversitarioInformado

${ }^{3}$ Que combina más de un modo semiótico, i.e. video: verbal, visual y sonoro.
} 
la función que cumplen estos significados en la conformación de: 1) una base epistémica: cómo son gestionados los conocimientos previos y nuevos sobre el conflicto estudiantil, sus antecedentes e impactos, etc., y; 2) una base ideológica: cómo se negocian las actitudes grupales acerca de las demandas del movimiento, sus formas de protesta, principios y valores, etc.

Primero agrupamos las entradas por cada tipo de género para descubrir patrones de significación transversales. Una vez cubiertas las entradas analizamos los comentarios asociados a cada una de ellas. Dado que el macrogénero posteo es habitualmente bimodal (tanto las entradas como los comentarios emplean recursos verbales y visuales), debimos establecer qué significados aportaba cada modo semiótico en su respectiva subunidad y luego dar cuenta de las relaciones intermodales existentes entre ellas (si estos significados resultaban convergentes o divergentes entre sí). Finalmente, agrupamos los géneros por cada uno de los espacios públicos-mediáticos considerados, de modo de dilucidar los procesos de transposición de significados de unos discursos y prácticas sociales a otras, es decir, su naturaleza transmedial.

\section{SíNTESIS DE RESULTADOS CUALITATIVOS}

A continuación sintetizamos los principales hallazgos que desprendemos hasta la fecha del análisis cualitativo, con el objetivo de aportar un modelo teórico-analítico que, a pesar de formularse sobre la base de un caso de estudio concreto, podría llegar a extrapolarse a otros movimientos que emplean las redes sociales dentro de sus prácticas mediales. Este modelo intenta dar cuenta tanto de los procesos de significación como de las prácticas discursivas más prominentes que llevan a cabo los/as estudiantes en UI. Entendemos que procesos y prácticas se basan en un principio de co-construcción, en la medida en que los primeros abarcan patrones subyacentes a la producción de sentidos que se estabilizan a lo largo de las movilizaciones, mientras que las segundas dan cuenta de las funciones sociales, políticas y cognitivas de los géneros multimodales que se difunden con mayor regularidad y transversalidad en el tiempo.

\section{a) Procesos de construcción de significados}

\section{i) Oposición dialógica en redes sociales}

La oposición es la condición esencial que configura las disputas ideológicas, cuya organización se basa en el esquema nosotros v/s ellos (Van Dijk, 1999). De esta manera, sus usuarios/as -en cuanto miembros posicionados diferencialmente dentro del conflicto en curso- pueden desarrollar distintos tipos de disenso discursivo, orientándose al fortalecimiento de acciones, objetivos, recursos, identidades, normas y valores del endogrupo, y al subsecuente debilitamiento del exogrupo y sus componentes.

La estructura de comunicación de Facebook involucra una dinámica estable de publicación e interacción a dos niveles interdependientes. De un lado, las entradas se utilizan, habitualmente, para producir y/o difundir discursos tendientes a legitimar los atributos de los/estudiantes movilizados/as y a deslegitimar los atributos de las autoridades políticas, configurándose modos de representación grupal competitivos. De otro lado, los 
comentarios se utilizan, habitualmente, para refrendar o desestimar tales representaciones, con argumentos y evaluaciones que activan lógicas de socialización y discusión política propias de la comunidad online.

La finalidad de este proceso de oposición es redefinir públicamente el sentido del conflicto educativo, negociando el conocimiento social disponible sobre las movilizaciones y sus actores críticos. De ello resulta un tipo de subversión ideológica basado en un principio dialógico explícito (Lemke, 2003), de modo que tanto las tácticas de ataque como de contestación promueven la construcción de significados que ambos grupos definen como mutuamente excluyentes en función de sus intereses socio-políticos. En este contexto, comunidades online como UI operan sintetizando dichos significados a partir de los contenidos de las publicaciones que gestionan, resguardando que la confrontación que motiva el conflicto sea continuamente enmarcada y reenmarcada por sus seguidores/as. Con ello se modela un tipo de oposición mediatizada que se regenera y consolida a medida que avanza el ciclo movilizatorio, lo que le permite a los/as estudiantes configurar esquemas de interpretación colectiva por fuera de las constricciones comunicativas que imponen otros lugares de interlocución formal con el poder.

\section{ii) Resignificación en redes sociales}

Este proceso aborda los rasgos lingüísticos y semióticos de estas formas de oposición. Concretamente, atiende tres fenómenos de significación que resultan prominentes en redes sociales: a) la intermedialidad: que trata de las relaciones entre distintas plataformas de comunicación, lo que implica que cuando se accede a los discursos sobre el movimiento estudiantil ni estos ni las referencias que les subyacen se derivan de un único medio (Lehtonen, 2000); b) la hipermodalidad: que caracteriza la naturaleza multimodal de los recorridos hipertextuales en la web (Lemke, 2003) en tanto los/as usuarios/as desarrollan rutas de navegación indefinidas (Lemke, 2009a), y; c) la intertextualidad: que puede ser de dos tipos, horizontal cuando incluye vínculos más o menos manifiestos entre textos primarios provenientes de un medio en particular, y vertical, cuando dichos textos son asociados a otros textos secundarios disponibles en entornos online $\mathrm{u}$ offline, los cuales aluden explícita o implícitamente a los primeros (Lehtonen, 2000).

Estos procesos afectan transversalmente la producción y la interpretación discursiva en la Web Social, en general, y en la comunidad de UI, en particular, generando una experiencia transmediática (Lemke, 2009b) en virtud de la cual se enlaza, extrae y negocia el contenido social, político y cultural de sus publicaciones conjuntamente. Con ello, los/as estudiantes pueden reconstruir significados sobre el conflicto educativo, combinando medios y modos comunicativos con gran complejidad y sofisticación. Estas condiciones facilitan, además, que los/as jóvenes actúen como creadores de discursos resistentes o contradiscursos (Cárdenas, 2014a), gestionando discrecionalmente los recursos lingüísticosemióticos disponibles para construir sus propios mensajes. Por lo anterior, la forma en que se representa la acción colectiva juvenil en los espacios público-mediáticos ya no queda supeditada únicamente a los discursos de actores ajenos al movimiento, pudiendo proponer y disputar los sentidos conferidos a sus demandas y motivaciones continuamente.

Lo anterior tiene, a lo menos, dos tipos de rendimientos relevantes. Por un lado, esta resignificación deriva de la multiplicación creativa de los mecanismos de expresión juvenil, 
lo que robustece la elaboración y la propagación de toda clase de textos que aseguran la visibilización de su protesta, tales como fotografías, vídeos, afiches, grafitis, etc. Estos productos expanden y articulan diversos referentes simbólicos de la lucha estudiantil, y ayudan a revalidar sus acciones en soportes simultáneos de comunicación e interacción. Por otro lado, la resignificación es posible sólo en la medida en que se generan impactos sociocognitivos concretos en las formas de conocer, identificarse y alinearse ideológicamente con el movimiento. De esta manera, tanto la producción como la articulación de discursos propios y ajenos persiguen potenciar representaciones sociales distintas de aquellas que proponen las elites políticas y mediáticas que negativizan la acción colectiva estudiantil. Dichas redefiniciones se emplazan y amplifican estratégicamente, de modo que los procesos de fijación de sentidos en torno al conflicto educativo dejan de ser potestad de unos pocos. Las redes sociales, en esta dirección, provocan la apertura a modos contrahegemónicos de significación.

iii) Transcontextualización en redes sociales

Este proceso supone que la producción discursiva se imbrica, a su vez, con las posibilidades de diseño, difusión y recepción provistas por la arquitectura de la Web Social. Así, se observa que muchas de las prácticas de resignificación llevadas a cabo por los/as jóvenes operan bajo una lógica de resemiotización (Iedema, 2003), toda vez que las comunidades estudiantiles online transponen y reformulan sus saberes, memorias y posicionamientos políticos cuando interconectan sentidos desde unos contextos mediáticos a otros (Cárdenas, 2014a). Estas relaciones se fundan en un principio de inconmensurabilidad (Lemke, 2005), a partir del cual el potencial semiótico de un modo comunicativo es contextualizado transversalmente en virtud de las características del medio tecnológico en el que aparecen los discursos.

Este proceso se expresa, concretamente, mediante la selección y la reutilización de contenidos propios y ajenos en UI. Ya sea que se trate de discursos que legitiman o deslegitiman la acción política juvenil, cuando éstos son tomados y trasladados desde su lugar de origen para hacerlos circular dentro de la comunidad online, sus sentidos primarios se modifican en el nuevo contexto en el que aparecen, activando otros excedentes simbólicos. Este desplazamiento explica varios fenómenos de significación incongruente, como los que se derivan, por ejemplo, de usos humorísticos, satíricos o irónicos, especialmente cuando se transcontextualizan discursos que construyen representaciones estereotipadas sobre el movimiento estudiantil. Este proceso también puede dar cuenta de otros fenómenos de significación derivados de la mixtura o remezcla de discursos provenientes de distintas fuentes, los cuales pueden ocasionar efectos transgresores o polémicos cuando tensionan representaciones disímiles sobre el conflicto educativo.

La transposición de significados a través de múltiples medios provoca que los/as usuarios/as reacomoden sucesivamente sus marcos interpretativos en virtud de las interacciones en las que se involucran, en la medida en que pueden actualizar un conjunto de conocimientos previos, recuerdos, opiniones y actitudes que, habitualmente, se articulan con las de otros/as usuarios/as de la Web Social. En este sentido, el proceso de transcontextualización también implica un trabajo socio-cognitivo específico, pues permite explorar "cómo y por qué los significados que hacemos aquí y ahora están conectados a los 
realizados por otros con los que formamos una red de interdependencia mutua" (Lemke, 2001: 80). Es, precisamente, el carácter móvil y fluido de los discursos 2.0 (Herring, 2013) lo que permite innovar en sus usos tecno-políticos, de maneras que subvierten los patrones convencionales de emisión y propagación.

\section{b) Prácticas discursivas}

Los procesos de construcción de significados descritos están a la base de dos prácticas discursivas que hemos investigado hasta aquí (Cárdenas, 2016, 2014a, 2014b, en prensa). La primera práctica que describimos es distintiva de los meses en que irrumpen las movilizaciones, aun cuando acaba asentándose y permanece durante los años posteriores, perdiendo centralidad, y se distingue porque recurre casi exclusivamente a discursos que son ajenos al movimiento. La segunda práctica se vuelve notoria una vez que el ciclo movilizatorio ha experimentado cierto grado de maduración, esto es, cuando los/as estudiantes avanzan de modalidades contestatarias a otras más creativas. Su principal característica consiste en incluir un mayor y mejor manejo de producciones propias.

\section{i) Prácticas de gestión contrainformativa}

Las interacciones comunicativas en redes sociales configuran un espacio mediático estructurado por flujos informativos y contrainformativos: de un lado, se hallan los medios controlados por el duopolio político-empresarial, a cuyo cargo está la mayor parte de los portales de noticias del país; de otro lado, se ubican las comunidades estudiantiles online que seleccionan, reponen y evalúan dichas noticias con el fin de abrir el debate motivando los comentarios de sus seguidores/as. Por lo tanto, esta práctica abarca usos asociados con la reubicación y la discusión de informaciones provenientes mayoritariamente de la prensa tradicional, lo cual, a corto andar, deviene en la formulación de contradiscursos que buscan cuestionar estos mensajes oficiales.

La reposición noticiosa se constituye en una macro unidad de análisis compuesta por tres etapas funcionales: un segmento textual (usualmente el titular o la bajada de la noticia) que es publicado de manera literal, el hipervínculo que redirige al medio de donde se extrae y los comentarios o respuestas que desencadena. Dicha reposición ocurre bajo criterios de impacto y economía, dado que se eligen sólo ciertos fragmentos de noticias cuyos contenidos usualmente construyen representaciones polémicas sobre la acción política del movimiento, opción que puede interpretarse en virtud de la asignación de relevancia que efectúan los/as administradores de las comunidades estudiantiles online para determinar aquellas publicaciones que pueden generar mayores reacciones (diálogo, valoraciones y viralización) por parte de sus seguidores/as.

En este sentido, la gestión de contenidos repuestos responde a un macropropósito comunicativo nuclear: disentir de los significados construidos por los discursos mediáticos hegemónicos, mediante la insinuación de finalidades veladas o sesgos sistemáticos. Con ello, los/as estudiantes actualizan las posiciones de poder en la comunicación online pues, aun cuando se les confiera un lugar subordinado en los medios tradicionales, en el espacio de negociación política conformado por sus redes sociales dichos roles se subvierten, equiparando su influencia. 
Esta práctica activa dinámicas de interacción que, al no depender ni estar supeditadas a las esferas adultas y poderosas, minimizan los efectos manipuladores de las elites político-mediáticas, por un lado, y profundizan los sentidos de pertenencia de los/as jóvenes movilizados/as, por el otro. Con ello, las comunidades estudiantiles online devienen en lugares de oposición y resignificación de los discursos periodísticos predominantes, delineándose como contramedios que reapropian informaciones importantes para el fortalecimiento y la continuidad de las movilizaciones. Su uso favorece la formación de una conciencia crítica, en virtud de la cual los/as estudiantes gestionan estratégicamente el conocimiento asociado a sus luchas y sus causas.

\section{ii) Prácticas de expansión transmedia}

Con el devenir de las movilizaciones estudiantiles se observa progresivamente la utilización de discursos de elaboración propia ${ }^{4}$. Su particularidad reside en que estos pueden evocar interesantes relaciones entre sí, aludiendo a temáticas, referentes o símbolos comunes, cuyas representaciones convergen y se expanden continuamente a través de la Web Social (Cárdenas, 2014a). De esta forma, los/as jóvenes construyen ciertos nodos de significación que son trabajados en el tiempo, y que sirven principalmente a los fines de enmarcado para la acción y la identificación colectiva.

Estos discursos se caracterizan por explotar diversas modalidades semióticas en combinación, patentes en productos como vídeos, afiches, memes, caricaturas y fotografías de lienzos, rayados e intervenciones callejeras que visibilizan los repertorios de acción estudiantil en espacios público-mediáticos simultáneos (Cárdenas, 2014b). Así, los discursos juveniles circulan de unos lugares a otros - de la calle a las redes sociales-, y de unas prácticas sociales a otras -de las convocatorias online a las manifestaciones públicas-, constituyendo una estrategia de alto impacto mediático en razón de la cual los procesos de transcontextualización de significados aseguran la mayor notoriedad posible de su protesta.

A partir de la correlación de diferentes publicaciones es posible seguir o, más concretamente, leer determinadas cadenas semánticas que son transversales a los discursos multimodales que replican y amplifican por las redes sociales sentidos relevantes para la representación de la acción política juvenil. Esta práctica de expansión transmedia se activa habitualmente a partir de ciertas coyunturas noticiosas ${ }^{5}$ que, a la larga, tienen el potencial de convertirse en hitos comunicacionales para el movimiento, los cuales se incorporan al reservorio de la memoria estudiantil modelando sus acciones futuras.

Los significados que se expanden por la Web Social conforman recursos privilegiados para configurar narraciones a gran escala, las cuales resultan esenciales en los procesos socio-cognitivos que estimulan la pertenencia grupal, reparando especialmente en las creencias y las emociones que soportan y orientan el enfrentamiento ideológico. De este modo, se negocian continuamente representaciones sociales críticas sobre el movimiento estudiantil, las cuales se constituyen en base a otras representaciones que han sido

\footnotetext{
${ }^{4}$ Dado que es complejo determinar fehacientemente la autoría de los textos que circulan en redes sociales, cuando se alude a textos propios se considera en un sentido amplio a aquellos cuyos contenidos auto-representan la acción política juvenil desde la perspectiva del endogrupo, contando sus productores/as como miembros o aliados/as.

${ }^{5}$ Por ejemplo, un caso de expansión transmedia se configura a partir de la denominación "inútiles subversivos" (Cárdenas 2014a), empleada por el ex senador de derecha Carlos Larraín en las declaraciones que ofrece a la prensa tras la marcha no autorizada del 4 de agosto de 2011, una de las más emblemáticas del movimiento estudiantil.
} 
previamente difundidas por actores y medios diversos. De allí que las redes sociales operen como emplazamientos de importantes síntesis intersubjetivas, con alcances simbólicos y materiales aún por dimensionar.

\section{CONCLUSIONES}

Los/as jóvenes, al integrar y combinar las plataformas de la Web Social en sus repertorios de acción e interacción comunicativa, impactan, a lo menos, en tres niveles complementarios:

- socio-semiótico: vinculado con las distintas formas de explotar múltiples recursos expresivos en la producción, reapropiación y difusión de mensajes propios y ajenos, reivindicando con ello su posición de interlocutores legítimos en el debate educativo;

- socio-cognitivo: relacionado con la redefinición de los marcos de interpretación que circunscriben sus identidades grupales y sus posicionamientos ideológicos, fortaleciendo así sus dinámicas de disenso y afiliación; y;

- político-histórico: asociado con la convergencia de espacios de resistencia online y offline, cuestión que releva la centralidad de las oportunidades mediáticas que los/as estudiantes gestionan progresivamente según las necesidades de representación social del movimiento.

De esta manera, los usos contrainformativos y transmediales llevados a cabo por los/as estudiantes en redes sociales les ayudan a posicionar y expandir sus ejercicios de oposición y resignificación, mediante la transcontextualización de discursos y prácticas sociales de unos espacios público-mediáticos a otros. Este fenómeno obedece a la evolución del movimiento estudiantil reciente (2006-2016), en la medida en que sus modalidades de protesta van satisfaciendo funciones pragmáticas y semánticas sensibles a las condiciones contextuales en las que se desenvuelven, es decir, se adecúan y responden a las oportunidades políticas que operan a lo largo del conflicto educativo en la última década.

En este sentido, el movimiento estudiantil se instituye como un movimiento social reflexivo (Martín Rojo, 2016a), en tanto sus usos tecno-políticos van consolidándose como un bien estratégico para la consecución de sus luchas y sus causas colectivas. Es, precisamente, la construcción de espacios de discursividad interrelacionados lo que permite a los/as jóvenes mover, simultáneamente, el horizonte de lo decible, lo pensable y lo posible (Cárdenas, 2014b).

SANTIAGO (CHILE), MAYO 2016

RECIBIDO: MAYO 2016 ACEPTADO: SEPTIEMBRE 2016

\section{REFERENCIAS BIBLIOGRÁFICAS}


AGUILERA, ÓSCAR (2014): Generaciones: Movimientos juveniles, políticas de identidad y disputas por la visibilidad en el Chile neoliberal. Buenos Aires: CLACSO.

(2012): "Repertorios y ciclos de movilización juvenil en Chile (2000-2012)". Utopía y Praxis Latinoamericana Vol. 17, № 57. Maracaibo: Universidad del Zulia.

(2010a): "Acción colectiva juvenil: De movidas y finalidades de adscripción". Nómadas No 32. Bogotá: Universidad Central.

(2010b): "Cultura política y política de las culturas juveniles”. Utopía y Praxis Latinoamericana Vol. 15, No 50. Maracaibo: Universidad del Zulia.

AGUIRRE, FÉLIX Y ÓSCAR GARCÍA (2015): "Más allá de malestar. Una hipótesis sociológica sobre el significado político del movimiento estudiantil chileno". Revista de Sociologia e Política Vol. 23, No 53. Curitiba: Universidade Federal do Paraná.

ALCALDE, IGNASI (2015): Visualización de la información. Barcelona: UOC Ediciones.

ARANCIBIA, CRISTINA, STEPHEN SADLIER Y LÉSMER MONTECINO (2016): "Impact of social media on Chilean student movement". En P. Kirsi, S. Mills y T. Skelton (editores): Politics, citizenship and rights. Singapore: Springer Science+Business Media.

BAEZA-YATES, RICARDO (2009): “Tendencias en minería de datos de la web”. El Profesional de la Información Vol. 18, $\mathrm{N}^{\circ} 1$. Barcelona: EPI. (2005): "Excavando la web". El Profesional de la Información Vol. 13, № 1. Barcelona: EPI.

BELL, DAVID (2006): Cybercultures: Cyberculture, cyberpolitics, cybersociety. New York-London: Routledge.

BELLEI, CRISTIÁN, CRISTIAN CABALIN Y VÍCTOR ORELLANA (2014): “The 2011 Chilean student movement against neoliberal education policies". Studies in Higher Education Vol. 39, № 3. London: Taylor \& Francis.

Y CRISTIAN CABALIN (2013): "Chilean student movements: Sustained struggle to transform a market-oriented educational system". Current Issues in Comparative Education Vol. 15, № 2. New York: Columbia University.

BENNETT, W. LANCE Y ALEXANDRA SEGERBERG (2013): The logic of connective action. Cambridge: Cambridge University Press.

BUTLER, MARY (2011): Clicktivism, slacktivism, or 'real' activism? Cultural codes of American activism in the Internet era. Ann Arbor: Pro Quest.

CABALIN, CRISTIAN (2014): "Estudiantes conectados y movilizados: El uso de Facebook en las protestas estudiantiles en Chile". Comunicar Vol. 22, No 43. Huelva: Grupo Comunicar.

(2012): "Neoliberal Education and Student Movements in Chile: Inequalities and Malaise". Policy Futures in Education No 10. Oxford: Symposium Journals.

CABELLO, CRISTIAN (2012): "Poner el cuerpo en duda. Interrupciones políticas mediatizadas en Chile". Sociedad y Equidad $\mathrm{N}^{\mathrm{o}} 3$. Santiago: Universidad de Chile.

CAMMAERTS, BART, ALICE MATTONI Y PATRICK MCCURDY (EDITORES) (2013): Mediation and protest movements. Bristol-Chicago: Intellect.

(2012): "Protest logics and the mediation opportunity structure". European Journal of Communication Vol. 27, N 2. London: Sage.

CÁRDENAS, CAMILA (2016): "Representación de la acción política juvenil en redes sociales: Análisis crítico de las prácticas discursivas producidas durante las 
movilizaciones estudiantiles en Chile (2011-2013)". Revista Austral de Ciencias Sociales $N^{\circ}$ 30. Valdivia: Universidad Austral.

(2014a): "Inútiles y subversivos: Representación transmedia de los estudiantes chilenos en redes sociales". Romanica Olomucensia Vol. 26, $\mathrm{N}^{\mathrm{o}}$ 2. Olomuc: Univerzita Palackého v Olomouci.

(2014b): "Representación de la acción política de los estudiantes chilenos. Movilización de significados en redes sociales". Última Década Vol. 22, No 40. Valparaíso: CIDPA.

(2014c): "Representación visual de la movilización estudiantil en Chile: Las fotografías de las marchas como espacios de narración, actuación e identificación política". Onomázein $\mathrm{N}^{\mathrm{o}}$ Esp. IX ALSFAL. Santiago: Pontificia Universidad Católica.

(2014d): “Jóvenes e (in)visibilización histórica: Modos de representación ideológica de la juventud chilena en el pasado reciente (1970-1990)". Signos Vol. 47, No 85. Valparaíso: Pontificia Universidad Católica.

(2011): “(In)visibilización juvenil: Acerca de las posibilidades de las y los jóvenes en la historia reciente del país". Última Década Vol. 19, №35. Valparaíso: CIDPA.

(en prensa). "Representación online del movimiento estudiantil chileno: Reapropiación noticiosa en Facebook”. Estudios Filológicos. Valdivia: Universidad Austral.

CASTELlS, MANUEL (2014): "El poder de las redes". Vanguardia Dossier No 50. Barcelona: La Vanguardia Ediciones.

(2012): Redes de indignación y esperanza. Madrid: Alianza. (2009): Comunicación y poder. Madrid: Alianza.

(2007): "Communication, power and counter-power in the network society". International Journal of Communication Vol. 1. Los Angeles: USC Annenberg Press.

CONDEZA, RAYÉN (2009): "Las estrategias de comunicación utilizadas por los adolescentes". Cuadernos de Información Vol. 24. Santiago: Pontificia Universidad Católica.

COSTA, PAOLA (2008): El rol de los Fotologs en el movimiento estudiantil chileno de mayo 2006. Tesis presentada para obtener el grado de Máster Erasmus Mundus en Ingeniería de Medios para la Educación. Poitiers-Lisboa-Madrid: Universidad de Poitiers-Universidad Técnica de Lisboa-Universidad Nacional de Educación a Distancia.

COSTANZA-CHOCK, SASHA (2014): Out of the shadows, into the streets! Transmedia organizing and the immigrant rights movement. Cambridge-London: MIT Press.

(2013): "Transmedia mobilization in the Popular Association of the Oaxacan Peoples, Los Angeles". En B. Cammaerts, A. Mattoni y P. McCurdy (editores): Mediation and protest movements. Bristol-Chicago: Intellect.

(2012): "Mic check! Media cultures and the Occupy Movement". Social Movement Studies Vol. 11, No 3-4. London-New York: Routledge.

CUADRA, ÁLVARO (2012): "Cultura de la protesta: Protesta de la cultura". América Latina en Movimiento [online]. Recuperado el 31 de marzo de 2015 de: http://www.alainet.org/es/active/57119 
DOMEDEL, ANDREA Y MACARENA PEÑA Y LILLO (2008): El mayo de los pingüinos. Santiago: Ediciones Radio Universidad de Chile.

EGGINS, SUSAN (2002): Introducción a la lingüística sistémica. La Rioja: Servicio de Publicaciones Universidad de La Rioja.

FAIRCLOUGH, NORMAN (1995): Critical discourse analysis: The critical study of language. London-New York: Longman.

FEIXA, CARLES, ARIADNA FERNÁNDEZ-PLANELLS Y MÓNICA FIGUERAS-MAZ (2016): "Generación Hashtag. Los movimientos juveniles en la era de la web social". Revista Latinoamericana de Ciencias Sociales, Niñez y Juventud Vol. 14, $\mathrm{N}^{\mathrm{o}} 1$. Manizales: Universidad de Manizales-CINDE.

, INÊS PEREIRA Y JEFFREY JURIS (2009): "Global citizenship and the 'new, new' social movements: Iberian connections". Young Vol. $17 \mathrm{~N}^{\circ}$ 4. London: Sage.

FERNÁNDEZ, ROBERTO (2013): "El espacio público en disputa: Manifestaciones políticas, ciudad y ciudadanía en el Chile actual". Psicoperspectivas Vol. 12, No 2. Valparaíso: Pontificia Universidad Católica.

FERNÁNDEZ-PLANELLS, CARLES FEIXA Y MÓNICA FIGUERAS-MAZ (2013): “15$\mathrm{M}$ en España: Diferencias y similitudes en las prácticas comunicativas con los movimientos previos". Última Década Vol. 21 N 39. Valparaíso: CIDPA.

GAMSON, WILLIAM Y GADI WOLFSFELD (1993): "Movements and media as interacting systems". Annals of the American Academy of Political and Social Science Vol. 526. London: Sage.

GARCÍA, ÓSCAR Y FÉLIX AGUIRRE (2016): "Spatial practices and narratives: The GenkiDama for Education by Chilean students". En L. Martín Rojo (editora): Occupy. The spatial dynamics of discourse in global protest movements. AmsterdamPhiladelphia: John Benjamins.

GARRETÓN, MANUEL ET AL. (2011): "Movimiento social, nuevas formas de hacer política y enclaves autoritarios. Los debates del Consejo Asesor para la Educación en el gobierno de Michelle Bachelet en Chile". Polis. Revista Latinoamericana Vol. 10 N$^{\circ}$ 30. Santiago: Universidad de Los Lagos-CISPO.

GEORGALOU, MARIZA (2015): "Small stories of the Greek crisis on Facebook". Social Media + Society Vol 1, No 2. London: Sage.

HALLIDAY, MICHAEL Y CHRISTIAN MATTHIESSEN (2014): Halliday's introduction to functional grammar ( $4^{\text {th }}$ Ed.). London-New York: Routledge.

HERRING, SUSAN (2013): "Discourse in Web 2.0: Familiar, reconfigured, and emergent". En D. Tannen y A. M. Trester (editoras): Discourse 2.0: Language and new media. Washington DC: Georgetown University Press.

HILL, KEVIN Y JOHN HUGHES (1998): Cyberpolitics: Citizen activism in the age of the Internet. New York: Rowman \& Littlefield.

JORDAN, TIM Y PAUL TAYLOR (2004): Hacktivism and cyberwars: Rebels with a cause? London-New York: Routledge.

KRESS, GÜNTHER (2010): Multimodality: A social semiotic approach to contemporary communication. London-New York: Routledge.

Y THEO VAN LEEUWEN (2006): Reading images. The grammar of visual design. London-New York: Routledge. 
Última DÉCADA N²45, ProYeCTO JUVENTUDES, DiCIEMBRE 2016, PP. 93-116

(2001): Multimodal discourse: The modes and media of contemporary communication. Oxford: Oxford University Press.

LATORRE, JUAN IGNACIO (2013): "Moviment estudiantil: Re-polititzant la societat xilena". Educació Social No 55. Barcelona: Universitat Ramon Llull.

LEHTONEN, MIKKO (2000): “On no man's land. Theses on intermediality”. Nordicom Information Vol. 3-4. Göteborg: University of Gothenburg.

LEMKE, JAY (2009a): "Transmedia traversals: Marketing meaning and identity". En A. Baldry y E. Montagna (editores): Interdisciplinary perspectives on multimodality: Theory and practice. Campobasso: Palladino.

(2009b): "Multimodality, identity, and time". En C. Jewitt (editora): Routledge handbook of multimodal analysis. London: Routledge.

(2005): "Critical analysis across media: Games, franchises, and the new cultural order". En M. Labarta Postigo (editora): Approaches to critical discourse analysis. Valencia: Universidad de Valencia.

(2003): "Travels in hypermodality". Visual Communication Vol. 1, No 3. London: Sage.

(2001): "Discursive technologies and the social organization of meaning". Folia Linguistica Vol. $35 \mathrm{~N}^{\mathrm{o}} 1-2$. Berlin: De Gruyter.

MARTIG, SERGIO ET AL. (2003): “Un modelo unificado de visualización”. Actas del $9^{\circ}$ Congreso Argentino de Ciencias de la Computación. La Plata: CACIC.

MARTIN, JAMES (2014): "Evolving systemic functional linguistics: Beyond the clause". Functional Linguistics Vol. $1 \mathrm{~N}^{\mathrm{o}}$ 3. Berlin: Springer.

MARTÍN ROJO, LUISA (EDITORA) (2016a): Occupy. The spatial dynamics of discourse in global protest movements. Amsterdam-Philadelphia: John Benjamins.

(2016b): "Taking over the square: The role of linguistic practices in contesting urban spaces". En L. Martín Rojo (editora): Occupy. The spatial dynamics of discourse in global protest movements. Amsterdam-Philadelphia: John Benjamins.

(2013): "Paisajes lingüísticos de indignación. Prácticas comunicativas para tomar las plazas". En S. Aguilar (editor): Anuario del conflicto social 2012. Barcelona: Observatorio del Conflicto Social-Universidad de Barcelona.

MATTONI, ALICE (2013): "Repertoires of communication in social movement processes". En B. Cammaerts, A. Mattoni y P. McCurdy (editores): Mediation and protest movements. Bristol-Chicago: Intellect.

MAYOL, ALBERTO (2012): El derrumbe del modelo. Santiago: LOM.

Y CARLA AZÓCAR (2011): "Politización del malestar, movilización social y transformación ideológica: El caso "Chile 2011"”. Polis. Revista Latinoamericana Vol. 10, No 30. Santiago: Universidad de Los Lagos-CISPO.

MCADAM, DOUG, JOHN MCCARTHY Y MAYER ZALD (1999): “Oportunidades, estructuras de movilización y procesos enmarcadores: Hacia una perspectiva sintética y comparada de los movimientos sociales". En D. McAdam, J. McCarthy y M. Zald (editores): Movimientos sociales: Perspectivas comparadas. Madrid: Itsmo.

MCCAUGHEY, MARTHA Y MICHAEL AYERS (2003): Cyberactivism: Online activism in theory and practice. New York-London: Routledge. 
MELUCCI, ALBERTO (1994): “¿Qué hay de nuevo en los nuevos movimientos sociales?” En E. Laraña y J. Gusfield (coordinadores): Los nuevos movimientos sociales. Madrid: CIS.

MILLALEO, SALVADOR (2011): "La ciberpolítica de los movimientos sociales en Chile: Algunas reflexiones y experiencias". Anales Vol. $7 \mathrm{~N}^{\circ}$ 2. Santiago: Universidad de Chile.

MONTESANO, NICOLINA Y ESPERANZA MORALES (2015): "Multimodal narrative as an instrument for social change: Reinventing democracy in Spain-The case of 15 M". Critical approaches to discourse analysis across disciplines Vol. $7 \mathrm{~N}^{\circ} 2$. Lancaster: Lancaster University.

MUÑOZ, VÍCTOR (2011): "Juventud y política en Chile: Hacia un enfoque generacional”. Última Década Vol. 19 No 35. Valparaíso: CIDPA.

PEÑA-LÓPEZ, ISMAEL (2013): "Casual politics: Del clicktivismo a los movimientos emergentes y el reconocimiento de patrones". Educación Social Vol. 55. Barcelona: Universitat Ramon Llull.

PICKERILL, JENNY (2003): Cyberprotest: Environmental activism online. Manchester: Manchester University Press.

PINO-OJEDA, WALESCKA (2014): "Insurgency of discourse and affective intervention: The Chilean students' movement”. Argos Aotearoa Vol. 1. Auckland: SG Digital.

PORTILLO, MARICELA ET AL. (2012): “De la generación X a la generación@. Trazos transicionales e identidades juveniles en América Latina”. Última Década Vol. 20, № 37. Valparaíso: CIDPA.

ROMANO, MANUELA (2015): "La protesta social como 'laboratorio' de creatividad metafórica". Discurso \& Sociedad Vol. 9 N 1-2. Barcelona: D\&S.

SAMUEL, ALEXANDRA (2004): Hacktivism and the future of political participation. Cambridge: Harvard University.

SCHERMAN, ANDRÉS Y ARTURO ARRIAGADA (2012a): “Jóvenes, postmaterialismo y consumo de medios". En A. Scherman (editor): Jóvenes, participación y medios 2011. Santiago: Feedback-Ediciones Universidad Diego Portales.

(2012b): "Disposition to vote and media consumption patterns among Chilean youth". Communication \& Society Vol. 25, No 1. Navarra: University of Navarra.

Y SEBASTIÁn VALENZUELA (2013): "La protesta en la era de las redes sociales: El caso chileno". En A. Arriagada y P. Navia (editores): Intermedios. Medios de comunicación y democracia en Chile. Santiago: Ediciones Universidad Diego Portales.

(2014): "Student and environmental protests in Chile: The role of social media". Politics Vol. $35 \mathrm{~N}^{\mathrm{o}}$ 2. London: Sage.

SIERRA, SONIA (2012): "Humor y crítica social en la red en el entorno del 15M". Discurso \& Sociedad Vol. 6, N 3. Barcelona: D\&S.

SOMMA, NICOLÁS (2012): "The Chilean student movement of 2011-2012: Challenging the marketization of education". Interface Vol. $4 \mathrm{~N}^{\mathbf{2}}$ 2. Maynooth: National University of Ireland.

TARROW, SIDNEY (2011): El poder en movimiento. Los movimientos sociales, la acción colectiva y la política. Madrid: Alianza. 
TASCÓN, MARIO Y YOLANDA QUINTANA (2012): Ciberactivismo. Las nuevas revoluciones de las multitudes conectadas. Madrid: Los Libros de la Catarata.

TILLY, CHARLES Y LESLEY WOOD (2010): Los movimientos sociales, 1768-2008. Desde sus orígenes a Facebook. Barcelona: Crítica. (2002): "Repertorios de acción contestaria en Gran Bretaña: 1758-1834”. En M. Traugott (editor): Protesta social. Repertorios y ciclos de acción colectiva. Barcelona: Hacer.

TORET, JAVIER (COORDINADOR) (2015): Tecnopolítica y 15M: La potencia de las multitudes conectadas. Barcelona: UOC Ediciones. ET AL. (2013): Tecnopolítica: La potencia de las multitudes conectadas. Barcelona: UOC Ediciones.

TORRES, RODRIGO Y PAOLA COSTA (2012): "Uso e impacto de las redes sociales de Internet sobre las movilizaciones juveniles en Chile: ¿Hacia nuevas formas de organización colectiva?”. En M. D. Souza, P. Cabello y C. del Valle (editores): Medios, edades y culturas. Temuco: Universidad de La Frontera.

VALDERRAMA, LORENA (2013): "Jóvenes, ciudadanía y tecnologías de información y comunicación. El movimiento estudiantil chileno". Revista Latinoamericana de Ciencias Sociales, Niñez y Juventud Vol. $11 \mathrm{~N}^{\mathrm{o}}$ 1. Manizales: Universidad de Manizales-CINDE.

VALENZUELA, SEBASTIÁN (2013): "Unpacking the use of social media for protest behavior: The roles of information, opinion expression, and activism". American Behavioral Scientist Vol. 57, No 7. London: Sage.

(2012): "La protesta en la era de Facebook: Manifestaciones juveniles y uso de redes sociales en Chile 2009-2011”. En A. Scherman (editor): Jóvenes, participación y medios 2011. Santiago: Feedback-Ediciones Universidad Diego Portales.

VAN DE DONK, WIM ET AL. (2004): Cyberprotest: New media, citizens, and social movements. New York-London: Routledge.

VAN DIJK, TEUN (2014): Discourse and knowledge. Cambridge: Cambridge University Press.

(2012): Discurso y contexto. Barcelona: Gedisa.

(2011a): "Introduction: The study of discourse". En T. Van Dijk (editor): Discourse studies. A multidisciplinary introduction ( $2^{\text {nd }} E d$.). London: Sage.

(2011b): Sociedad y discurso. Barcelona: Gedisa.

(1999): Ideología. Barcelona: Gedisa.

VAN LEEUWEN, THEO (2008): Discourse and practice: New tools for critical discourse analysis. Oxford: Oxford University Press. (2005): Introducing social semiotics. London-New York: Routledge.

WODAK, RUTH (2013): “Critical discourse analysis - Challenges and perspectives". En R. Wodak (editora): Critical discourse analysis (Vol. I). London: Sage. 